\title{
Controllability of Neutral Impulsive Differential Inclusions with Non-Local Conditions
}

\author{
Dimplekumar N. Chalishajar', Falguni S. Acharya ${ }^{2 *}$ \\ ${ }^{1}$ Department of Mathematics and Computer Science, Virginia Military Institute (VMI), \\ Lexington, USA \\ ${ }^{2}$ Department of Applied Sciences Humanities, Institute of Technology and Management Universe, \\ Vadodara, India \\ E-mail: *falguni69@yahoo.co.in,dipu17370@yahoo.com,chalishajardn@vmi.edu \\ Received July 14, 2011; revised September 27, 2011; accepted October 7, 2011
}

\begin{abstract}
In this short article, we have studied the controllability result for neutral impulsive differential inclusions with nonlocal conditions by using the fixed point theorem for condensing multi-valued map due to Martelli [1]. The system considered here follows the P.D.E involving spatial partial derivatives with $\alpha$-norms.
\end{abstract}

Keywords: Controllability, Neutral Impulsive Differential Inclusions, Spatial Partial Derivative, Martelli Fixed Point Theorem

\section{Introduction}

In this paper we have discussed the controllability of nonlocal Cauchy problem for neutral impulsive differential inclusions of the form

$$
\left\{\begin{aligned}
& \frac{\mathrm{d}}{\mathrm{d} t}\left[x(t)-F\left(t, x\left(h_{1}(t)\right)\right)\right] \in A x(t)+B u(t)+G\left(t, x\left(h_{2}(t)\right)\right) ; \\
& t \in J:=[0, b] ; t \neq t_{k} \\
&\left.\Delta x\right|_{t=t_{k}}=I_{k}\left(x\left(t_{k}^{-}\right)\right) ; k=1,2, \cdots m ; x(0)+g(x)=x_{0} \in X
\end{aligned}\right.
$$

where the linear operator $(-A)$ generates an analytic semigroup $\{T(t)\}_{t>0} ; G$ is a multi-valued map and

$\Delta x_{t=t_{k}}=x\left(t_{k}^{+}\right)-x\left(t_{k}^{-}\right), x\left(t_{k}^{+}\right)=\lim _{h \rightarrow 0^{+}} x\left(t_{k}+h\right)$ and $x\left(t_{k}^{-}\right)=\lim _{h \rightarrow 0^{+}} x\left(t_{k}-h\right)$ represent the right and left limits of $x(t)$ at $t=t_{k}$ respectively, $x_{0} \in X$,

$F: J \times X \rightarrow P(X) \backslash \phi$ is a multi-valued map [ $P(X)$ is the family of all subsets of $\mathrm{X}]$ and $g \in C(J, X)$. Also the control function $u \in L^{2}(J, U)$, a Banach space of admissible control functions with $\mathrm{U}$ as a Banach space. $\mathrm{B}$ is a bounded linear operator from $U$ to $X$ and $X$ is a separable Banach space with norm $\|\cdot\|$.

$I_{k}: X \rightarrow \overline{D(A)} ; k=1,2, \cdots, m$ and $h_{1}, h_{2} \in C(J, J)$.

As a model we consider the following system of heat equations;

$$
\left\{\begin{array}{l}
\frac{\partial}{\partial t}\left[z(t, x)-F(t, z(\cos t, x)), \frac{\partial z}{\partial x}(\cos t, x)\right] \\
=\frac{\partial^{2} z(t, x)}{\partial x^{2}}+u(t, x)+G\left(t, z(\cos t, x), \frac{\partial z}{\partial x}(\cos t, x)\right) \\
z(t, 0)=z(t, \pi)=0 \\
z\left(t_{k}^{+}\right)-z\left(t_{k}^{-}\right)=I_{k}\left(z\left(t_{k}^{-}\right)\right) \\
t \neq t_{k} ; k=1,2, \cdots, m \\
z(0, x)+g(z(t, x))=z_{0}(x), 0 \leq x \leq \pi, t \in[0,1]
\end{array}\right.
$$

Since $F$ and $G$ involve spatial partial derivative, the results obtained by other authors cannot be applied to our system even if $g()=$.0 . This is the main motivation of this paper.

The existence and controllability of the following system is studied by Benchohra and Ntouyas [2]

$$
\left\{\begin{array}{l}
\frac{\mathrm{d}}{\mathrm{d} t}\left[x(t)-g\left(t, x_{t}\right)\right] \in A x(t)+B u(t)+F\left(t, x_{t}\right) ; \\
\quad t \in J:=[0, b] ; t \neq t_{k} \\
\left.\Delta x\right|_{t=t_{k}}=I_{k}\left(x\left(t_{k}^{-}\right)\right) ; k=1,2, \cdots, m ; x(t)=\phi(t), t \in(-\infty, 0) .
\end{array}\right.
$$

Here authors have proved exact controllability by using fixed point theorem for condensing multi-valued maps due to Martelli. In this paper, we have discussed controllability results with $\alpha$-norms as in [3] with de- 
viating arguments in terms involving spatial partial derivatives.

As indicated in [4], and reference therein, the nonlocal Cauchy problem $x(0)+g(x)=x_{0}$ can be applied in different fields with better effect than the classical initial condition $x(0)=x_{0}$. For example in [5], the author described the diffusion phenomenon of a small amount of gas in a transparent tube by using the formula

$$
g(x)=\sum_{i=0}^{p} c_{i} x\left(t_{i}\right)
$$

where $c_{i}, i=0,1, \cdots, p$ are given constants and $0<t_{0}, t_{1}, \cdots, t_{p}<b$. In this case the above equation allows the additional measurement at $t_{i}, i=0,1, \cdots, p$. In the past several years theorems about controllability of differential, integro-differential, fractional differential systems and inclusions with nonlocal conditions have been studied by Chalishajar and Acharya [6-9], Benchohra and Ntouyas [10,11], and Hernandez, Rabello and Henriquez [12] and the references therein. In [13], Chalishajar discussed exact controllability of third order nonlinear integro-differential dispersion system without compactness of semigroup.

Xianlong Fu and Yueju Cao [14], has discussed the existence of mild solution for neutral partial differential inclusions involving spatial partial derivative with $\alpha$ norms in Banach space. However in their work authors impose some severe assumptions on the operator family generator by $(-A)$, i.e. $(-A): D(A) \subset X \rightarrow X$ is an infinitesimal generator of a compact analytic semigroup of a uniformly bounded linear operator $\{T(t)\}_{t \geq 0}$, which imply that underlying space $X$ has finite dimension and so the example considered in [14], and subsequently in Section 4 is ordinary differential equation but not partial differential equation which shows lack of existence (exact controllability) in abstract (control) system (refer [15]). This fact and several other applications of neutral equation (inclusions) are the main motivation of this paper.

In Section 3 (followed by Preliminaries) of present paper we discuss the controllability of neutral impulsive differential inclusion with nonlocal condition with deviating arguments with $\alpha$-norm, which is the generalization of [14], in a finite dimensional space. The example is given in Section 4 to support the theory. In Section 5 we study exact controllability of same system in infinite dimension space by dropping the compactness assumption of semigroup $\{T(t)\}_{t \geq 0}$. Here we generalized the result proved in Section 3.

\section{Preliminaries}

In this section, we shall introduce some basic definitions, notations and lemmas which are used throughout this paper.

Let $(X,\|\cdot\|)$ be a Banach space. $C(J, X)$ is the Banach space of continuous functions from $J$ into $X$ with the norm defined by

$$
\|x\|_{J}:=\sup \{\|x(t)\|: t \in J\} .
$$

Let $B(X)$ be the Banach space of bounded linear operators from $X$ into $X$ with standard norm

$$
\|N\|_{B(X)}:=\sup \{\|N(x)\|:\|x\|=1\} .
$$

A measurable function $x: J \rightarrow X$ is Bochner integrable if and only if $\|x\|$ is Lebesgue integrable. (For properties of the Bochner integral see [16]). Let $L^{1}(J, X)$ denotes the Banach space of Bochner integrable functions $x: J \rightarrow X$ with norm

$\|x\|_{L^{1}}=\int_{0}^{b}\|x(t)\| \mathrm{d} t$ for all $x \in L^{1}(J, X)$.

We use the notations $P(X)=\left\{Y \in 2^{X}: Y \neq \phi\right\}$, $P_{c l}(X)=\{Y \in P(X): Y$ closed $\}$,

$P_{b}(X)=\{Y \in P(X):$ Ybounded $\}$,

$P_{c}(X)=\{Y \in P(X): Y$ convex $\}$, and

$P_{c p}(X)=\{Y \in P(X):$ Ycompact $\}$.

A multi-valued map $G: X \rightarrow 2^{X}$ is convex (respectively closed) valued if $G(x)$ is convex (respectively closed) for all $x \in X$.

The map $G$ is bounded on bounded sets if $G(B)=\cup_{x \in B} G(x)$ is bounded in $X$ for any bounded set $B$ of $X$. (i.e. $\left.\sup _{x \in B}\{\sup \{\|x\|: x \in G(x)\}\}<\infty\right)$.

$G$ is called upper semi-continuous (u.s.c.) on $X$ if for each $x_{0} \in X$, the set $G\left(x_{0}\right)$ is a nonempty closed subset of $X$ and if for each open set $B$ of $X$ containing $G\left(x_{0}\right)$, there exists an open neighborhood $A$ of $x_{0}$ such that $G(A) \subseteq B$.

The map $G$ is said to be completely continuous if $G(B)$ is relatively compact for every bounded subset $B \subseteq X$.

If the multi-valued map $G$ is completely continuous with nonempty compact values, then $G$ is u.s.c. if and only if $G$ has a closed graph, That is, if

$x_{n} \rightarrow x_{0}, y_{n} \rightarrow y_{0}$, where $y_{n} \in G\left(x_{n}\right)$ then $y_{0} \in G\left(x_{0}\right)$. $G$ has a fixed point if there is $x \in X$ such that $x \in G(x)$.

A multi-valued map $G: J \rightarrow B C C(X)$ is said to be measurable, if for each $x \in X$, the distance function $Y: J \rightarrow R$ defined by

$$
Y(t)=d(x, G(t))=\inf \{\|x-z\|: z \in G(t)\}
$$

is measurable.

An upper semi-continuous map $G: X \rightarrow 2^{X}$ is said to be condensing, if for any bounded subset $B \subseteq X$, with $\alpha(B) \neq 0$, we have $\alpha(G(B))<\alpha(B)$, where $\alpha$ denotes the Kuratowski measure of non-compactness. 
We remark that a completely continuous multi-valued map is the easiest example of a condensing map. For more details on multivalued maps see the books of Deimling [17].

Throughout this paper, $A: D(A) \subset X \rightarrow X$ will be the infinitesimal generator of a compact analytic semigroup of uniformly bounded linear operator $T(t)$. Let $0 \in \rho(A)$, then it is possible to define the fractional power $A^{\alpha}$, for $0 \leq \alpha \leq 1$, as a closed linear operator on its domain $D\left(A^{\alpha}\right)$. Furthermore, the subspace $D\left(A^{\alpha}\right)$ is dense in $\mathrm{X}$ and the expression

$$
\|x\|_{\alpha}=\left\|A^{\alpha} x\right\| ; x \in D\left(A^{\alpha}\right)
$$

defines a norm on $D\left(A^{\alpha}\right)$. Hereafter we denote by $X_{\alpha}$, the Banach space $D\left(A^{\alpha}\right)$ normed with $\|x\|_{\alpha}$. Then for each $0<\alpha \leq 1, X_{\alpha}$ is a Banach space, and $X_{\alpha} \longmapsto X_{\beta}$ for $0<\beta<\alpha \leq 1$ and the imbedding is compact whenever the resolvent operator of $A$ is compact.

Semigroup $\{T(t)\}_{t \geq 0}$ satisfies the following properties:

a) there is a $M \geq 1$ such that

$$
\|T(t)\| \leq M \text { for all } 0 \leq t \leq \alpha ;
$$

b) for any $0<\alpha \leq 1$, there exists a positive constant $C_{\alpha}$ such that

$$
\left\|A^{\alpha} T(t)\right\| \leq \frac{C_{\alpha}}{t^{\alpha}} ; 0<t \leq \alpha
$$

For more details about the above preliminaries, we refer to $([18,19])$.

In order to define the solution of the system (1) we shall consider the space

$$
\Omega=\left\{x:[0, b] \rightarrow X_{\alpha} ; x_{k} \in C\left(J_{k}, X_{\alpha}\right) ; k=0,1, \cdots, m\right.
$$

and there exist $x\left(t_{k}^{-}\right)$and $x\left(t_{k}^{+}\right) ; k=0,1, \cdots, m$

$$
\text { with } \left.x\left(t_{k}^{-}\right)=x\left(t_{k}\right), x(0)+g(x)=x_{0}\right\} \text {, }
$$

which is a Banach space with the norm

$$
\|x\|_{\Omega}=\max \left\{\left\|x_{k}\right\|_{J_{k}} ; k=0,1, \cdots, m\right\}
$$

where $x_{k}$ is the restriction of $x$ to

$J_{k}=\left(t_{k}, t_{k+1}\right], k=0,1, \cdots, m$ and $\left\|x_{k}\right\|_{J_{k}}=\sup _{s \in J_{k}}\left\|x_{k}(s)\right\|_{\alpha}$.

For the system (1) we assume that the following hypotheses are satisfied for some $\alpha \in(0,1)$ :

(H1) Let $W: L^{2}(J, U) \rightarrow X_{\alpha}$ be the linear operator defined by

$$
W u=\int_{0}^{b} T(b-s) B u(s) \mathrm{d} s
$$

The $W: L^{2}(J, U) /$ kerW $\rightarrow X_{\alpha}$ induces a bounded invertible operator $\tilde{W}^{-1}$ and there exists positive constant $M_{1}$ and $M_{2}$ such that and $\|B\| \leq M_{1}$ and
$\left\|\tilde{W}^{-1}\right\| \leq M_{2}$.

(H2) i) there exists a constant $\beta \in(0,1)$ such that $F:[0, b] \times X_{\alpha} \rightarrow X_{\beta}$ is a continuous function, and $A^{\beta} F:[0, b] \times X_{\alpha} \rightarrow X_{\beta}$ satisfies the Lipschitz condition, that is, there exists a constant $L>0$ such that

$$
\left\|A^{\beta} F\left(t_{1}, x_{1}\right)-A^{\beta} F\left(t_{2}, x_{2}\right)\right\|_{\alpha} \leq L\left(\left|t_{1}-t_{2}\right|+\left\|x_{1}-x_{2}\right\|_{\alpha}\right),
$$

for any $0 \leq t_{1}, t_{2} \leq b ; x_{1}, x_{2} \in X_{\alpha}$.

ii) Moreover, there exists a constant $L_{1}>0$ such that the inequality

$$
\left\|A^{\beta} F(t, x)\right\|_{\alpha} \leq L_{1}\left(\|x\|_{\alpha}+1\right),
$$

holds for any $x \in X_{\alpha}$.

(H3) The multi-valued map $G: J \times X_{\alpha} \rightarrow P_{c, c p}(X)$ satisfies the following conditions:

i) for each $t \in J$, the function $G(t,):. X_{\alpha} \rightarrow P_{c, c p}(X)$ is u.s.c. and for each $x \in X_{\alpha}$, the function

$G(., x): J \rightarrow P_{c, c p}(X)$ is measurable. Also for each fixed $y \in \Omega$ the set

$$
S_{G, x}=\left\{v \in L^{1}(J, X): v(t) \in G\left(t, x\left(h_{2}(t)\right)\right) \text { fora.e. } t \in J\right\}
$$

is nonempty.

ii) for each positive number $l \in N$, there exists a positive function $w(l)$ dependent on $l$ such that

$$
\sup _{\|x\| \leq l}\|G(t, x)\| \leq w(l)
$$

and $\liminf _{l \rightarrow \infty} \frac{w(l)}{l}=\gamma<\infty$ where

$\|G(t, x)\|=\sup \{\|v\|: v \in G(t, x)\},\|x\|_{\alpha}=\sup _{0 \leq s \leq \alpha}\|x(s)\|_{\alpha}$.

(H4) $h_{i} \in C(J, J), i=1,2 . g: \Omega \rightarrow X_{\alpha}$ is continuous and satisfies that

i) there exists positive constants $L_{2}$ and $L_{2^{\prime}}$ such that

$$
\|g(y)\|_{\alpha} \leq L_{2}\|y\|_{\Omega}+L_{2}^{\prime} \text { for all } y \in \Omega .
$$

ii) $A^{\alpha} g$ is completely continuous map.

(H5) $I_{k} \in C\left(X_{\alpha}, X_{\alpha}\right), k=1,2, \cdots, m$, are all bounded, that is, there exist constants $d_{k}, k=1,2, \cdots, m$, such that

$$
\left\|I_{k}(x)\right\|_{\alpha} \leq d_{k}, \text { for each } x \in X_{\alpha} .
$$

Now we define the mild solution for the system (1).

DEFINITION 2.1 The system (1) is said to be nonlocally controllable on the interval $J$ if for every $x(0)+g(x) \in D(A)$ and $x_{0}, z_{1} \in X$, there exists a control $u \in L^{2}\left(0, b: L^{2}(0,2 \pi)\right)=L^{2}(J, U)$ such that the corresponding solution $x(\cdot)$ of $(1)$ satisfies

i) $x(b)+g(x)=z_{1}$ with $x(0)+g(x)=x_{0}$;

ii) $\left.\Delta x\right|_{t=t_{k}}=I_{k}\left(x\left(t_{k}^{-}\right)\right) ; k=1,2, \cdots, m$;

iii) there exists a function $v \in L^{1}(J, X)$ such that 


$$
\begin{aligned}
v(t) \in G( & \left.t, x\left(h_{2}(t)\right)\right) \quad \text { a.e. on } J \text { and } \\
x(t)= & T(t)\left[x_{0}-g(x)-F\left(0, x\left(h_{1}(0)\right)\right)\right]+F\left(t, x\left(h_{1}(t)\right)\right) \\
& +\int_{0}^{t} A T(t-s) F\left(s, x\left(h_{1}(s)\right)\right) \mathrm{d} s+\int_{0}^{t} T(t-s) v(s) \mathrm{d} s \\
& +\int_{0}^{t} T(t-s)(B u)(s) \mathrm{d} s \\
& +\sum_{0<t_{k}<t} T\left(t-t_{k}\right) I_{k}\left(x\left(t_{k}^{-}\right)\right) ; t \in J, v \in S_{G, x} .
\end{aligned}
$$

The following lemmas are crucial in the proof of our main theorem.

LEMMA 2.2 [20] Let $X$ be a Banach space. Let $G: J \times X \rightarrow P_{b, c l, c}(X)$ satisfies that

i) For each $x \in X,(t, x) \mapsto G(t, x)$ is measurable with respect to $t$ and for each $t \in J,(t, x) \rightarrow G(t, x)$ is u.s.c. with respect to $\mathrm{x}$.

ii) For each fixed $x \in C(J, X)$, the set $S_{G, x}=\left\{v \in L^{1}(J, X): v(t) \in G\left(t, x\left(h_{2}(t)\right)\right)\right.$, for a.e. $\left.t \in J\right\}$ is nonempty.

Let $\Gamma$ be a linear continuous mapping from $L^{1}(J, X)$ to $C(J, X)$ then the operator $\Gamma \operatorname{OS}_{G}: C(J, X) \rightarrow P_{c p, c}(C(J, X))$, $x \rightarrow\left(\Gamma O S_{G}\right)(x):=\Gamma\left(S_{G, x}\right)$ is a closed graph operator in $C(J, X) \times C(J, X)$.

LEMMA 2.3 [17] Let $\Omega$ be a bounded and convex set in Banach space $X . \quad F: \Omega \rightarrow 2^{\Omega} \backslash \phi$ be an upper semi-continuous and condensing multi-valued map. If for every $x \in \Omega, F(x)$ is closed and convex set in $\Omega$, then $F$ has a fixed point in $\Omega$.

\section{Controllability Result}

We are now able to state and prove our main controllability result.

THEOREM 3.1 Let $x_{0} \in X_{\alpha}$. If the hypotheses (H1)(H5) are satisfied, then the system (1) is controllable provided

$$
\begin{aligned}
& L_{0}:=L\left[(M+1) M_{0}+\frac{1}{\beta} C_{1}-\beta \alpha^{\beta}\right]<1 \\
& \left(M_{0} L_{1}+L_{2}\right) M+M_{0} L_{1} \\
& +\frac{1}{\beta} C_{1}-\beta \alpha^{\beta} L_{1}+\frac{1}{(1-\alpha)} C_{\alpha} \alpha^{1-\alpha} \gamma<1
\end{aligned}
$$

and

$$
\begin{aligned}
M_{3}= & \left\|z_{1}\right\|_{\alpha}+M_{1}\left\|x_{0}\right\|_{\alpha}+(M+1)\left(L_{2} l+L_{2}^{\prime}\right) \\
& +(M+1) M_{0} L_{1}(l+1)+\frac{C_{1-\beta}}{\beta} L_{1}(l+1) b^{\beta} \\
& +\frac{C_{\alpha}}{1-\alpha} w(l) b^{1-\alpha}+M \sum_{k=1}^{m} d_{k},
\end{aligned}
$$

where, $M_{0}=\left\|A^{-\beta}\right\|$.

Proof. Let $C:=C(J, \overline{D(A)})$ denote the Banach space of continuous functions from $J$ to $\overline{D(A)}$ normed by

$$
\|x\|_{C}=\sup \{|x(t)|: t \in J\}
$$

Using hypothesis (H5) for an arbitrary function $x($.) and $z_{1} \in \overline{D(A)}$ define the control

$$
\begin{aligned}
u_{x}(t)= & \tilde{W}^{-1}\left[z_{1}-g(x)-T(b)\left[x_{0}-g(x)-F\left(0, x\left(h_{1}(0)\right)\right)\right]\right. \\
& -F\left(b, x\left(h_{1}(b)\right)\right)+\int_{0}^{b} A T(b-s) F\left(s, x\left(h_{1}(s)\right)\right) \mathrm{d} s \\
& \left.+\int_{0}^{b} T(b-s) v(s) \mathrm{d} s+\sum_{0<t_{k}<t} T\left(t-t_{k}\right) I_{k}\left(x\left(t_{k}^{-}\right)\right)\right]
\end{aligned}
$$

Using the above control, define a multi-valued map $N: \Omega \rightarrow 2^{\Omega}$ by

$$
\begin{aligned}
N(x)=\{y & \in \Omega: y(t)=T(t)\left[x_{0}-g(x)-F\left(0, x\left(h_{1}(0)\right)\right)\right] \\
& +F\left(t, x\left(h_{1}(t)\right)\right)+\int_{0}^{t} A T(t-s) F\left(s, x\left(h_{1}(s)\right)\right) \mathrm{d} s \\
& +\int_{0}^{t} T(t-s) v(s) \mathrm{d} s+\int_{0}^{t} T(t-s)(B u)(s) \mathrm{d} s \\
& \left.+\sum_{0<t_{k}<t} T\left(t-t_{k}\right) I_{k}\left(x\left(t_{k}^{-}\right)\right) ; t \in J, v \in S_{G, x}\right\} .
\end{aligned}
$$

By assumption on $F, g, I_{k}$ and the fact that $x_{0} \in X_{\alpha}$, it is obvious that $y(t) \in X_{\alpha}$.

Clearly the fixed points of $N$ are mild solutions to (1). We shall show that $\mathrm{N}$ satisfies the hypotheses of Lemma 2.3. The proof will be given in several steps. Step 1: There exists a positive number $l \in N$ such that $N\left(H_{l}\right) \subset H_{l}$, where

$$
H_{l}=\left\{x \in \Omega:\|x(t)\|_{\alpha} \leq l, 0 \leq t \leq \alpha\right\} \text {. }
$$

For each positive number $l, H_{l}$ is clearly a bounded closed convex set in $\Omega$. We claim that there exists a positive integer $l$ such that $N\left(H_{l}\right) \subset H_{l}$, where $N\left(H_{l}\right)=\cup_{x \in H_{l}} N(x)$. If it is not true, then for each positive integer $l$, there exist the functions $x_{l}(.) \in H_{l}$ and $y_{l} \in N\left(x_{l}\right)$, but $y_{l}(.) \notin H_{l}$, that is $\left\|y_{l}(t)\right\|_{\alpha}>l$ for some $t(l) \in[0, b]$, where $t(l)$ denotes $t$ is dependent on $l$. However on the other hand we have,

$$
\begin{aligned}
l<\left\|y_{l}(t)\right\|_{\alpha} & =\| T(t)\left[x_{0}-g\left(x_{l}\right)-F\left(0, x_{l}\left(h_{1}(0)\right)\right)\right] \\
& +F\left(t, x_{l}\left(h_{1}(t)\right)\right)+\int_{0}^{t} A T(t-s) F\left(s, x_{l}\left(h_{1}(s)\right)\right) \mathrm{d} s \\
& +\int_{0}^{t} T(t-s) v_{l}(s) \mathrm{d} s+\int_{0}^{t} T(t-s)\left(B u_{l}\right)(s) \mathrm{d} s \\
& +\sum_{0<t_{k}<t} T\left(t-t_{k}\right) I_{k}\left(x_{l}\left(t_{k}^{-}\right)\right) \|_{\alpha}, \text { where } v_{l} \in S_{G, x_{l}}
\end{aligned}
$$

Hence, 


$$
\begin{aligned}
l< & \left\|T(t)\left[x_{0}-g\left(x_{l}\right)-A^{-\beta} A^{\beta} F\left(0, x_{l}\left(h_{1}(0)\right)\right)\right]\right\|_{\alpha} \\
& +\left\|A^{-\beta} A^{\beta} F\left(t, x_{l}\left(h_{1}(t)\right)\right)\right\|_{\alpha} \\
& +\left\|\int_{0}^{t} A^{1-\beta} T(t-s) A^{\beta} F\left(s, x_{l}\left(h_{1}(s)\right)\right) \mathrm{d} s\right\|_{\alpha} \\
& +\left\|\int_{0}^{t} T(t-s) v_{l}(s) \mathrm{d} s\right\|_{\alpha}+\left\|\int_{0}^{t} T(t-s)\left(B u_{l}\right)(s) \mathrm{d} s\right\|_{\alpha} \\
& +\sum_{0<t_{k}<t}\left\|T\left(t-t_{k}\right)\right\|_{\alpha}\left\|I_{k}\left(x_{l}\left(t_{k}^{-}\right)\right)\right\|_{\alpha} \\
l \leq & M\left[\left\|x_{0}\right\|_{\alpha}+L_{2} l+L_{2}^{\prime}+M_{0} L_{1}(l+1)\right]+M_{0} L_{1}(l+1) \\
& +\int_{0}^{t} \frac{C_{1-\beta}}{(t-s)^{(1-\beta)}} L_{1}(l+1) \mathrm{d} s+\int_{0}^{t} \frac{C_{\alpha}}{(t-s)^{\alpha}} w_{1}(l) \mathrm{d} s \\
& +\int_{0}^{t} \frac{C_{\alpha}}{(t-s)^{\alpha}} M_{1} M_{2} M_{3} \mathrm{~d} s+M \sum_{k=1}^{m} d_{k}
\end{aligned}
$$

Dividing on both sides by $l$ and taking the lower limit as $l \rightarrow+\infty$ we get

$$
\left(M_{0} L_{1}+L_{2}\right) M+M_{0} L_{1}+C_{1-\beta} \frac{b^{\beta}}{\beta} L_{1}+C_{\alpha} \frac{b^{1-\alpha}}{1-\alpha} \gamma \geq 1 .
$$

This is a contradiction with Formula (2). Hence for some positive integer $N\left(H_{l}\right) \subseteq H_{l}$.

Step 2: $N(x)$ is convex for each $x \in \Omega$.

Indeed if $y_{1}, y_{2} \in N(x)$ then there exists $v_{1}, v_{2} \in S_{G, x}$ such that for each $t \in J$, we have

$$
\begin{aligned}
y_{i}(t)= & T(t)\left[x_{0}-g(x)-F\left(0, x\left(h_{1}(0)\right)\right)\right] \\
& +F\left(t, x\left(h_{1}(t)\right)\right)+\int_{0}^{t} A T(t-s) F\left(s, x\left(h_{1}(s)\right)\right) \mathrm{d} s \\
& +\int_{0}^{t} T(t-s) v_{i}(s) \mathrm{d} s+\int_{0}^{t} T(t-s)\left(B u_{i}\right)(s) \mathrm{d} s \\
& +\sum_{0<t_{k}<t} T\left(t-t_{k}\right) I_{k}\left(x\left(t_{k}^{-}\right)\right) ; i=1,2 .
\end{aligned}
$$

Let $0 \leq \lambda \leq 1$. Then for each $t \in J$ we have

$$
\begin{aligned}
& {\left[\lambda y_{1}+(1-\lambda) y_{2}\right](t)=T(t)\left[x_{0}-g(x)-F\left(0, x\left(h_{1}(0)\right)\right)\right]} \\
& \quad+F\left(t, x\left(h_{1}(t)\right)\right)+\int_{0}^{t} A T(t-s) F\left(s, x\left(h_{1}(s)\right)\right) \mathrm{d} s \\
& \quad+\int_{0}^{t} T(t-s)\left[\lambda v_{1}(s)+(1-\lambda) v_{2}(s)\right] \mathrm{d} s \\
& \quad+\int_{0}^{t} T(t-s) B\left[\lambda u_{1}(s)+(1-\lambda) u_{2}(s)\right] \mathrm{d} s \\
& \quad+\sum_{0<t_{k}<t} T\left(t-t_{k}\right) I_{k}\left(x\left(t_{k}^{-}\right)\right)
\end{aligned}
$$

Since $S_{G, x}$ is convex because $G$ has convex values, $\lambda y_{1}+(1-\lambda) y_{2} \in N(x)$.

Step 3: $N(x)$ is closed for each $x \in \Omega$.

Let $\left\{y_{n}\right\}_{n \geq 0} \in N(x)$ such that $y_{n} \rightarrow y$ in $\Omega$. Then $y \in \Omega$ and there exists $v_{n} \in S_{G, x}$ such that for every $t \in J$,

$$
\begin{aligned}
y_{n}(t)= & T(t)\left[x_{0}-g(x)-F\left(0, x\left(h_{1}(0)\right)\right)\right]+F\left(t, x\left(h_{1}(t)\right)\right) \\
& +\int_{0}^{t} A T(t-s) F\left(s, x\left(h_{1}(s)\right)\right) \mathrm{d} s \\
& +\int_{0}^{t} T(t-s) v_{n}(s) \mathrm{d} s+\int_{0}^{t} T(t-s)(B u)(s) \mathrm{d} s \\
& +\sum_{0<t_{k}<t} T\left(t-t_{k}\right) I_{k}\left(x\left(t_{k}^{-}\right)\right)
\end{aligned}
$$

Using the fact that $G$ has compact values, we may pass to a subsequence if necessary to get that $v_{n}$ conver-ges to $v \in L^{1}(J, X)$ and hence $v \in S_{G, x}$. Then for each $t \in J$,

$$
\begin{aligned}
& y_{n}(t) \rightarrow y(t)=T(t)\left[x_{0}-g(x)-F\left(0, x\left(h_{1}(0)\right)\right)\right] \\
& +F\left(t, x\left(h_{1}(t)\right)\right)+\int_{0}^{t} A T(t-s) \times F\left(s, x\left(h_{1}(s)\right)\right) \mathrm{d} s \\
& +\int_{0}^{t} T(t-s) v(s) \mathrm{d} s+\int_{0}^{t} T(t-s)(B u)(s) \mathrm{d} s \\
& +\sum_{0<t_{k}<t} T\left(t-t_{k}\right) I_{k}\left(x\left(t_{k}^{-}\right)\right) ; t \in J .
\end{aligned}
$$

Hence $y \in N(x)$.

Step 4: Next we show that the operator $N$ is u.s.c and condensing.

For this purpose, we decompose $N$ as $N=N_{1}+N_{2}$, where the operators $N_{1}, N_{2}$ are defined on $H_{l}$ respectively by

$$
\begin{aligned}
& \left(N_{1} x\right)(t)=F\left(t, x\left(h_{1}\right)(t)\right)-T(t) F\left(0, x\left(h_{1}(0)\right)\right. \\
& +\int_{0}^{t} A T(t-s) F\left(s, x\left(h_{1}(s)\right)\right) \mathrm{d} s \\
& N_{2} x=\left\{y \in \Omega: y(t)=T(t)\left[x_{0}-g(x)-F\left(0, x\left(h_{1}(0)\right)\right)\right]\right. \\
& +F\left(t, x\left(h_{1}(t)\right)\right)+\int_{0}^{t} A T(t-s) F\left(s, x\left(h_{1}(s)\right)\right) \mathrm{d} s \\
& +\int_{0}^{t} T(t-s) v(s) \mathrm{d} s+\int_{0}^{t} T(t-s)(B u)(s) \mathrm{d} s \\
& \left.+\sum_{0<t_{k}<t} T\left(t-t_{k}\right) I_{k}\left(x\left(t_{k}^{-}\right)\right) ; v \in S_{G, x}\right\} .
\end{aligned}
$$

We will verify that $N_{1}$ is a contraction while $N_{2}$ is a completely continuous operator.

To prove that $N_{1}$ is a contraction, we take $x_{1}, x_{2} \in H_{l}$ arbitrarily. Then for each $t \in J$ and by condition (H2), we have that

$$
\begin{aligned}
& \left\|\left(N_{1} x_{1}\right)(t)-\left(N_{1} x_{2}\right)(t)\right\|_{\alpha} \\
& \leq\left\|F\left(t, x_{1}\left(h_{1}(t)\right)\right)-F\left(t, x_{2}\left(h_{1}(t)\right)\right)\right\|_{\alpha} \\
& +\left\|T(t)\left[F\left(0, x_{1}\left(h_{1}(0)\right)\right)-F\left(0, x_{2}\left(h_{1}(0)\right)\right)\right]\right\|_{\alpha} \\
& +\left\|\int_{0}^{t} A T(t-s)\left[F\left(s, x_{1}\left(h_{1}(s)\right)\right)-F\left(s, x_{2}\left(h_{1}(s)\right)\right)\right] \mathrm{d} s\right\|_{\alpha} \\
& =\left\|A^{-\beta}\left[A^{\beta} F\left(t, x_{1}\left(h_{1}(t)\right)\right)-A^{\beta} F\left(t, x_{2}\left(h_{1}(t)\right)\right)\right]\right\|_{\alpha} \\
& +\| T(t) A^{-\beta}\left[A^{\beta} F\left(0, x_{1}\left(h_{1}(0)\right)-A^{\beta} F\left(0, x_{2}\left(h_{1}(0)\right)\right)\right] \|_{\alpha}\right. \\
& +\| \int_{0}^{t} A^{1-\beta} T(t-s) \\
& \quad \cdot\left[A^{\beta} F\left(s, x_{1}\left(h_{1}(s)\right)\right)-A^{\beta} F\left(s, x_{2}\left(h_{1}(s)\right)\right)\right] \mathrm{d} s \|_{\alpha} \\
& \leq\left[(M+1) M_{0} L+\int_{0}^{t} \frac{C_{1-\beta}}{(t-s)^{1-\beta}} L \mathrm{~d} s\right] . \sup _{0 \leq s \leq \alpha}\left\|x_{1}(s)-x_{2}(s)\right\|_{\alpha} \\
& \leq L\left[(M+1) M_{0}+\frac{1}{\beta} C_{1-\beta} \alpha^{\beta}\right] \sup _{0 \leq s \leq \alpha}\left\|x_{1}(s)-x_{2}(s)\right\|_{\alpha} \\
& =L_{0} \sup _{0 \leq s \leq \alpha}\left\|x_{1}(s)-x_{2}(s)\right\|_{\alpha}
\end{aligned}
$$


Thus, $\left\|N_{1} x_{1}-N_{2} x_{2}\right\|_{\alpha} \leq L_{0}\left\|x_{1}-x_{2}\right\|_{\alpha}$. Therefore by assumption $0<L_{0}<1, N_{1}$ is a contraction.

Next we show that $N_{2}$ is u.s.c. and condensing.

i) $N_{2}\left(H_{l}\right)$ is clearly bounded.

ii) $N_{2}\left(H_{l}\right)$ is equi-continuous.

Let $\tau_{1}, \tau_{2} \in J, \tau_{1}<\tau_{2}$. Let $x \in H_{l}$ and $y \in N_{2}(x)$. Then there exists $v \in S_{G, x}$ such that for each $t \in J$, we have

$$
\begin{aligned}
y(t) & =T(t)\left[x_{0}-g(x)\right]+\int_{0}^{t} T(t-s) v(s) \mathrm{d} s \\
& +\int_{0}^{t} T(t-s)(B u)(s) \mathrm{d} s+\sum_{0<t_{k}<t} T\left(t-t_{k}\right) I_{k}\left(x\left(t_{k}^{-}\right)\right)
\end{aligned}
$$

Then,

$$
\begin{aligned}
& \left\|y\left(\tau_{2}\right)-y\left(\tau_{1}\right)\right\|_{\alpha} \\
& \leq\left\|\left[T\left(\tau_{2}\right)-T\left(\tau_{1}\right)\right]\left(x_{0}-g(x)\right)\right\|_{\alpha} \\
& +\left\|\int_{0}^{\tau_{1}}\left[T\left(\tau_{2}-s\right)-T\left(\tau_{1}-s\right)\right] v(s) \mathrm{d} s\right\|_{\alpha}+\left\|\int_{\tau_{1}}^{\tau_{2}} T\left(\tau_{2}-s\right) v(s) \mathrm{d} s\right\|_{\alpha} \\
& +\| \int_{0}^{\tau_{1}}\left[T\left(\tau_{2}-s\right)-T\left(\tau_{1}-s\right)\right] B \tilde{W}^{-1} \\
& \quad \cdot\left\{z_{1}-g(x)-T(b)\left[x_{0}-g(x)-F\left(0, x\left(h_{1}(0)\right)\right)\right]\right. \\
& \quad-F\left(b, x\left(h_{1}(b)\right)\right)-\int_{0}^{b} A T(b-\eta) F\left(\eta, x\left(h_{1}(\eta)\right)\right) \mathrm{d} \eta \\
& \left.\quad-\int_{0}^{b} T(b-\eta) v(\eta) \mathrm{d} \eta-\sum_{0 \leq t_{k} \leq \tau_{1}} T\left(t-t_{k}\right) I_{k}\left(x\left(t_{k}^{-}\right)\right)\right\} \mathrm{d} s \|_{\alpha} \\
& +\| \int_{\tau_{1}}^{\tau_{2}} T\left(\tau_{2}-s\right) B \tilde{W}^{-1} \\
& \quad \cdot\left\{z_{1}-g(x)-T(b)\left[x_{0}-g(x)-F\left(0, x\left(h_{1}(0)\right)\right)\right]\right. \\
& \quad-F\left(b, x\left(h_{1}(b)\right)\right)-\int_{0}^{b} A T(b-\eta) F\left(\eta, x\left(h_{1}(\eta)\right)\right) d \eta \\
& \left.\quad-\int_{0}^{b} T(b-\eta) v(\eta) \mathrm{d} \eta+\sum_{\tau_{1} \leq t_{k} \leq \tau_{2}} T\left(t-t_{k}\right) I_{k}\left(x\left(t_{k}^{-}\right)\right)\right\} \mathrm{d} s \|_{\alpha}
\end{aligned}
$$

The right hand side tends to zero as $\left(\tau_{2}-\tau_{1}\right) \rightarrow 0$, since $T(t)$ is strongly continuous and the compactness of $\{T(t)\}_{t \geq 0}$ implies the continuity in the uniform operator topology. Thus $N_{2}\left(\right.$.) is equi-continuous on $H_{l}$.

iii) $\left(N_{2} H_{l}\right)(t)$ is relatively compact for each $t \in J$, where $\left(N_{2} H_{l}\right)(t)=\left\{y(t): y \in\left(N_{2} H_{l}\right)\right\}$.

Obviously, by condition $(H 4)(i i),\left(N_{2} H_{l}\right)(t)$ is relatively compact in $X_{\alpha}$ for $t=0$. Let $0<t \leq b$ be fixed and $0<\varepsilon<t$. For $x \in H_{l}$ and $y \in N_{2}(x)$, there exists a function $v \in S_{G, x}$ such that

$$
\begin{aligned}
y(t) & =T(t)\left[x_{0}-g(x)\right]+\int_{0}^{t-\varepsilon} T(t-s) v(s) \mathrm{d} s \\
& +\int_{t-\varepsilon}^{t} T(t-s) v(s) \mathrm{d} s+\int_{0}^{t-\varepsilon} T(t-s)(B u)(s) \mathrm{d} s \\
& +\int_{t-\varepsilon}^{t} T(t-s)(B u)(s) \mathrm{d} s+\sum_{0<t_{k}<t} T\left(t-t_{k}\right) I_{k}\left(x\left(t_{k}^{-}\right)\right)
\end{aligned}
$$

Define,

$$
\begin{aligned}
y_{\varepsilon}(t)= & T(t)\left[x_{0}-g(x)\right]+\int_{0}^{t-\varepsilon} T(t-s) v(s) \mathrm{d} s \\
& +\int_{0}^{t-\varepsilon} T(t-s)(B u)(s) \mathrm{d} s+\sum_{0<t_{k}<t} T\left(t-t_{k}\right) I_{k}\left(x\left(t_{k}^{-}\right)\right) \\
y_{\varepsilon}(t)= & T(t)\left[x_{0}-g(x)\right]+T(\varepsilon) \int_{0}^{t-\varepsilon} T(t-\varepsilon-s) v(s) \mathrm{d} s \\
& +T(\varepsilon) \int_{0}^{t-\varepsilon} T(t-\varepsilon-s)(B u)(s) \mathrm{d} s \\
& +\sum_{0<t_{k}<t} T\left(t-t_{k}\right) I_{k}\left(x\left(t_{k}^{-}\right)\right)
\end{aligned}
$$

Since $T(t)$ is compact, the set $Y_{\varepsilon}(t)=\left\{y_{\varepsilon}(t): y \in N_{2}\left(H_{l}\right)\right\}$ is relatively compact in $X_{\alpha}$ for every $\varepsilon, 0<\varepsilon<t$.

Moreover, for every $y \in N_{2}\left(H_{l}\right)$,

$$
\begin{aligned}
& \left\|y(t)-y_{\varepsilon}(t)\right\|_{\alpha} \\
& =\left\|\int_{t-\varepsilon}^{t} T(t-s) v(s) \mathrm{d} s\right\|_{\alpha}+\left\|\int_{t-\varepsilon}^{t} T(t-s)(B u)(s) \mathrm{d} s\right\|_{\alpha} \\
& \leq M \int_{t-\varepsilon}^{t} w(l) \mathrm{d} s \\
& +M M_{1} M_{2} \int_{t-\varepsilon}^{t}\left\{\left\|z_{1}\right\|_{\alpha}+\|g(x)\|_{\alpha}\right. \\
& +\|T(b)\|\left[\left\|x_{0}\right\|+\|g(x)\|+\left\|F\left(0, x\left(h_{1}(0)\right)\right)\right\|\right] \\
& +\left\|F\left(b, x\left(h_{1}(b)\right)\right)\right\| \\
& +\left\|\int_{0}^{b} A T(b-\eta) F\left(\eta, x\left(h_{1}(\eta)\right)\right) \mathrm{d} \eta\right\| \\
& +\left\|\int_{0}^{b} T(b-\eta) v(\eta) \mathrm{d} \eta\right\| \\
& \left.+\left\|\sum_{0<t_{k}<t} T\left(t-t_{k}\right) I_{k}\left(x\left(t_{k}^{-}\right)\right)\right\|\right\} \mathrm{d} s
\end{aligned}
$$

$\leq M w(l) \varepsilon$

$$
\begin{aligned}
& +M M_{1} M_{2} \int_{t-\varepsilon}^{t}\left\{\left\|z_{1}\right\|_{\alpha}+\left(L_{2} l+L_{2}^{\prime}\right)\right. \\
& +M\left[\left\|x_{0}\right\|+\left(L_{2} l+L_{2}^{\prime}\right)+M_{0} L_{1}(l+1)\right] \\
& +M_{0} L_{1}(l+1)+\int_{0}^{b} \frac{C_{1-\beta}}{(b-\eta)^{(1-\beta)}} L_{1}(l+1) \mathrm{d} \eta \\
& \left.\quad+\int_{0}^{b} \frac{C_{\alpha}}{(b-\eta)^{\alpha}} w(l) \mathrm{d} \eta+M \sum_{k=1}^{m} d_{k}\right\} \mathrm{d} s \\
& \leq M w(l) \varepsilon+M M_{1} M_{2} M_{3} \varepsilon \\
& \leq M \varepsilon\left[w(l)+M_{1} M_{2} M_{3}\right]
\end{aligned}
$$

Therefore, letting $\varepsilon \rightarrow 0$, we see that there are relatively compact sets arbitrarily close to the set $\left\{y(t): y \in N_{2}\left(H_{l}\right)\right\}$. Hence the set $\left\{y(t): y \in N_{2}\left(H_{l}\right)\right\}$ is relatively compact in $X_{\alpha}$.

As a consequence of (i),(ii), (iii) and together with the Arzela-Ascoli theorem we can conclude that $N_{2}: H_{l} \rightarrow 2^{H_{l}}$ is a completely continuous multi-valued map and, therefore, a condensing multi- valued map. 
iv) $N_{2}$ has a closed graph.

From the above steps we can see, for every $x \in H_{l}$, $N_{2}(x)$ is relatively compact and closed set, which can be testified as in Step 3. Hence $N_{2}(x)$ is a compact set.

Let $x_{n} \rightarrow x_{*}, \quad x_{n} \in H_{l}, y_{n} \in N_{2}\left(x_{n}\right)$ and $y_{n} \rightarrow y_{*}$. We must show that $y_{*} \in N_{2}\left(x_{*}\right) ; y_{n} \in N_{2}\left(x_{n}\right)$ means that there exists $v_{n} \in S_{G, x_{n}}$ such that, for each $t \in J$.

$$
\begin{aligned}
y_{n}(t) & =T(t)\left[x_{0}-g\left(x_{n}\right)\right]+\int_{0}^{t} T(t-s) v_{n}(s) \mathrm{d} s \\
& +\int_{0}^{t} T(t-s)\left(B u_{n}\right)(s) \mathrm{d} s+\sum_{0<t_{k}<t} T\left(t-t_{k}\right) I_{k}\left(x_{n}\left(t_{k}^{-}\right)\right)
\end{aligned}
$$

where

$$
\begin{aligned}
& u_{x_{n}}(t)=\tilde{W}^{-1}\left[z_{1}-g\left(x_{n}\right)-T(b)\left[x_{0}-g\left(x_{n}\right)-F\left(0, x\left(h_{1}(0)\right)\right)\right]\right. \\
& -F\left(b, x\left(h_{1}(b)\right)\right)+\int_{0}^{b} A T(b-s) F\left(s, x\left(h_{1}(s)\right)\right) \mathrm{d} s \\
& \left.+\int_{0}^{b} T(b-s) v_{n}(s) \mathrm{d} s+\sum_{0<t_{k}<t} T\left(t-t_{k}\right) I_{k}\left(x_{n}\left(t_{k}^{-}\right)\right)\right] \\
& \|\left(y_{n}(t)-T(t)\left[x_{0}-g\left(x_{n}\right)\right]-\int_{0}^{t} T(t-s)\left(B u_{n}\right)(s) \mathrm{d} s-\sum_{0<t_{k}<t} T\left(t-t_{k}\right) I_{k}\left(x_{n}\left(t_{k}^{-}\right)\right)\right) \\
& -\left(y_{*}(x)-T(t)\left[x_{0}-g\left(x_{*}\right)\right]-\int_{0}^{t} T(t-s)\left(B u_{*}\right)(s) \mathrm{d} s-\sum_{0<t_{k}<t} T\left(t-t_{k}\right) I_{k}\left(x_{*}\left(t_{k}^{-}\right)\right)\right) \|_{\Omega} \rightarrow 0 \text {, as } n \rightarrow \infty .
\end{aligned}
$$

Clearly, since $I_{k}, k=1,2, \cdots, m$ and $g$ are continu-

We must prove that there exists $v_{*} \in S_{G, x_{*}}$ such that

$$
\begin{aligned}
y_{*}(x)= & T(t)\left[x_{0}-g\left(x_{*}\right)\right]+\int_{0}^{t} T(t-s) v_{*}(s) \mathrm{d} s \\
& +\int_{0}^{t} T(t-s)\left(B u_{*}\right)(s) \mathrm{d} s+\sum_{0<t_{k}<t} T\left(t-t_{k}\right) I_{k}\left(x_{*}\left(t_{k}^{-}\right)\right)
\end{aligned}
$$

where

$$
\begin{aligned}
u_{x_{*}}(t) & \\
=\tilde{W}^{-1}[ & z_{1}-g\left(x_{*}\right)-T(b)\left[x_{0}-g\left(x_{*}\right)-F\left(0, x\left(h_{1}(0)\right)\right)\right] \\
& -F\left(b, x\left(h_{1}(b)\right)\right)+\int_{0}^{b} A T(b-s) F\left(s, x\left(h_{1}(s)\right)\right) \mathrm{d} s \\
& \left.+\int_{0}^{b} T(b-s) v_{*}(s) \mathrm{d} s+\sum_{0<t_{k}<t} T\left(t-t_{k}\right) I_{k}\left(x_{*}\left(t_{k}^{-}\right)\right)\right]
\end{aligned}
$$

From Lemma (H3) it follows that $\Gamma O S_{G}$ is a closed graph operator.

Moreover, we obtain that
Consider the linear continuous operator

$\Gamma: L^{1}(J, X) \rightarrow C(J, X), v \mapsto \Gamma(v)(t)=\int_{0}^{t} T(t-s) v(s) \mathrm{d} s$.

$$
\left(y_{n}(t)-T(t)\left[x_{0}-g\left(x_{n}\right)\right]-\int_{0}^{t} T(t-s)\left(B u_{n}\right)(s) \mathrm{d} s-\sum_{0<t_{k}<t} T\left(t-t_{k}\right) I_{k}\left(x_{n}\left(t_{k}^{-}\right)\right)\right) \in \Gamma\left(S_{G, x_{n}}\right) .
$$

Since $x_{n} \rightarrow x_{*}$, it follows from (H3) that

$$
y_{*}(t)-T(t)\left[x_{0}-g\left(x_{*}\right)\right] \int_{0}^{t} T(t-s)\left(B u_{*}\right)(s) \mathrm{d} s-\sum_{0<t_{k}<t} T\left(t-t_{k}\right) I_{k}\left(x_{*}\left(t_{k}^{-}\right)\right) \in \Gamma\left(S_{G, x_{*}}\right)
$$

that is, there must exist a $v_{*}(t) \in S_{G, x_{*}}$ such that

$$
\begin{aligned}
& y_{*}(t)-T(t)\left[x_{0}-g\left(x_{*}\right)\right] \\
& -\int_{0}^{t} T(t-s)\left(B u_{*}\right)(s) \mathrm{d} s-\sum_{0<t_{k}<t} T\left(t-t_{k}\right) I_{k}\left(x_{*}\left(t_{k}^{-}\right)\right) \\
& =\Gamma\left(v_{*}(t)\right)=\int_{0}^{t} T(t-s) v_{*}(s) \mathrm{d} s .
\end{aligned}
$$

Therefore, $N_{2}$ has a closed graph. Since $N_{2}$ is a completely continuous multi-valued map with compact value, $N_{2}$ is u.s.c. On the other hand $N_{1}$ is a contraction. Hence $N=N_{1}+N_{2}$ is u.s.c. and condensing.

By Lemma 2.3, there exists a fixed point $x($.) for $N$ on $H_{l}$. Therefore, the nonlocal Cauchy problem with impulsive effect (1) is controllable on $J$.

Particularly, if $G\left(t, x\left(h_{2}(t)\right)\right)$ is a single-valued map, then the system (1) will become

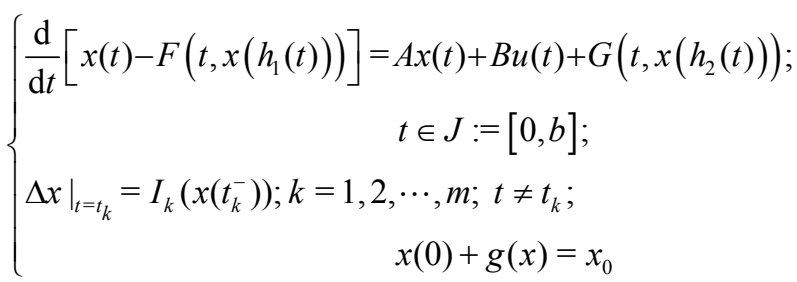

by using Sadovskii's fixed-point theorem for condensing map, we can analogously study the controllability of the system (4).

(H3)' The function $G: J \times X_{\alpha} \rightarrow X$ satisfies the following conditions:

i) for each $t \in J$, the function $G(t,):. X_{\alpha} \rightarrow X$ is 
continuous; and for each $x \in X_{\alpha}$, the function $G(., x): J \rightarrow X$ is strongly measurable.

ii) for each positive number $l \in N$, there exists a positive function $w(l)$ dependent on $l$ such that

$$
\sup \|G(t, x)\| \leq w(l) \text { and } \lim _{l \rightarrow \infty} \frac{w(l)}{l}=\gamma<\infty
$$

where

$$
\|x\|_{\alpha}=\sup _{0 \leq s \leq \alpha}\|x(s)\|_{\alpha} .
$$

THEOREM 3.2 Let $x_{0} \in X_{\alpha}$. If the hypotheses $(H 1),(H 2),(H 3)^{\prime},(H 4)$ and $(H 5)$ are satisfied, then the system (4) is controllable on $J$ provided (1), (2) and (1) hold.

Proof The mild solution of the system (4) is given by

$$
\begin{aligned}
x(t)= & T(t)\left[x_{0}-g(x)-F\left(0, x\left(h_{1}(0)\right)\right)\right] \\
& +F\left(t, x\left(h_{1}(t)\right)\right)+\int_{0}^{t} A T(t-s) F\left(s, x\left(h_{1}(s)\right)\right) \mathrm{d} s \\
& +\int_{0}^{t} T(t-s) G\left(s, x\left(h_{2}(s)\right) \mathrm{d} s+\int_{0}^{t} T(t-s)(B u)(s) \mathrm{d} s\right. \\
& +\sum_{0<t_{k}<t} T\left(t-t_{k}\right) I_{k}\left(x\left(t_{k}^{-}\right)\right) ; t \in J .
\end{aligned}
$$

We define the operator $N: \Omega \rightarrow \Omega$ by

$$
\begin{aligned}
(N x)(t)= & T(t)\left[x_{0}-g(x)-F\left(0, x\left(h_{1}(0)\right)\right)\right] \\
& +F\left(t, x\left(h_{1}(t)\right)\right)+\int_{0}^{t} A T(t-s) F\left(s, x\left(h_{1}(s)\right)\right) \mathrm{d} s \\
& +\int_{0}^{t} T(t-s) G\left(s, x\left(h_{2}(s)\right)\right) \mathrm{d} s+\int_{0}^{t} T(t-s)(B u)(s) \mathrm{d} s \\
& +\sum_{0<t_{k}<t} T\left(t-t_{k}\right) I_{k}\left(x\left(t_{k}^{-}\right)\right)
\end{aligned}
$$

Then we can decompose $N$ as $N=N_{1}+N_{2}$, where

$$
\begin{aligned}
\left(N_{1} x\right)(t)= & F\left(t, x\left(h_{1}\right)(t)\right)-T(t) F\left(0, x\left(h_{1}(0)\right)\right. \\
& +\int_{0}^{t} A T(t-s) F\left(s, x\left(h_{1}(s)\right)\right) \mathrm{d} s
\end{aligned}
$$

and

$$
\begin{aligned}
\left(N_{2} x\right)(t)= & T(t)\left[x_{0}-g(x)\right]+\int_{0}^{t} T(t-s) G\left(s, x\left(h_{2}(s)\right)\right) \mathrm{d} s \\
& +\int_{0}^{t} T(t-s)(B u)(s) \mathrm{d} s
\end{aligned}
$$

and verify that $N_{1}$ is a contraction while $N_{2}$ is a compact operator, thus Sadovskii's fixed-point theorem can be applied to the operator $N$ and hence $N$ has atleast a fixed point on $\Omega$, which shows that system (4) is controllable on the interval $J$. The desired proof is similar to Step 4 of Theorem 3.1.

\section{Example}

As an application of Theorem 3.2, we study the following impulsive partial function differential system with nonlocal condition

$$
\left\{\begin{array}{c}
\frac{\partial}{\partial t}\left[z(t, x)-\int_{0}^{\pi} b(t, y, x)\left[z(\sin t, y)+\frac{\partial z}{\partial y}(\sin t, y)\right] \mathrm{d} y\right] \\
=\frac{\partial^{2} z(t, x)}{\partial x^{2}}+u(t, y)+h\left(t, z(\sin t, x), \frac{\partial z}{\partial x}(\sin t, x)\right), \\
0 \leq t \leq 1,0 \leq x \leq \pi, t \neq t_{k}, k=1,2, \cdots, m ; \\
z(t, 0)=z(t, \pi)=0 ; z\left(t_{k}^{+}\right)-z\left(t_{k}^{-}\right)=I_{k}\left(z\left(t_{k}\right)\right), \\
k=1,2, \cdots, m \\
z(0, x)+\sum_{i=0}^{p} \int_{0}^{\pi} k_{i}(y, x) z\left(s_{i}, y\right) \mathrm{d} y=z_{0}(x), 0 \leq x \leq \pi
\end{array}\right.
$$

where $p$ is a positive integer, $0<s_{0}<s_{1}<\cdots<s_{p}<1$, and $0<t_{1}<t_{2}<\cdots t_{m}<\cdots<1 ; z_{0}(x) \in X=L^{2}([0, \pi]) . \quad A$ is defined by $A \omega=\omega^{\prime \prime} \quad$ with the domain

$$
\begin{gathered}
D(A)=H_{0}^{2}([0, \pi])=\{\omega(.) \in X: \omega, \\
\omega^{\prime} \text { are absolutely continuous, } \\
\left.\omega^{\prime \prime} \in X, \omega(0)=\omega(\pi)=0\right\}
\end{gathered}
$$

Then $A$ generates a strongly continuous semigroup $T($.) which is compact, analytic and self-adjoint.

a') Also A has a discrete spectrum representation

$$
A \omega=\sum_{n=1}^{\infty}\left(-n^{2}\right)<\omega, \omega_{n}>\omega_{n}, \omega \in D(A), n \in N ;
$$

where $\omega_{n}(x)=\sqrt{\frac{2}{\pi}} \sin (n x) ; n=1,2, \cdots \quad$ is the orthogonal set of eigenvector of $A$. The eigenvalues are $-n^{2}, n \in N$.

b') The operator $A^{\frac{1}{2}}$ is given by

$$
A^{\frac{1}{2}} \omega=\sum_{n=0}^{\infty} n<\omega, \omega_{n}>\omega_{n}
$$

on the space $D\left(A^{\frac{1}{2}}\right)=\left\{\omega(.) \in X: \sum_{n=1}^{\infty} n<\omega, \omega_{n}>\omega_{n} \in X\right\}$.

The control operator $B: L^{2}(J, X) \rightarrow X$ is defined by $(B u)(t)(y)=u(t, y) ; y \in(0, \pi)$ which satisfies condition (H5). Here $B$ is an identity operator and the control function $u($.$) is given in L^{2}([0, \pi], U)$.

We assume that the following conditions hold:

i) The function $b$ is measurable and

$$
\sup _{0 \leq t \leq 1} \int_{0}^{\pi} \int_{0}^{\pi} b^{2}(t, y, x) \mathrm{d} y \mathrm{~d} x<\infty .
$$

ii) The function $\frac{\partial^{2} b(t, y, x)}{\partial x^{2}}$ is measurable, $b(t, y, 0)=b(t, y, \pi)=0$, and

$$
N_{1}=\sup _{0 \leq t \leq 1}\left[\int_{0}^{\pi} \int_{0}^{\pi}\left(\frac{\partial^{2} b(t, y, x)}{\partial x^{2}}\right)^{2} \mathrm{~d} y \mathrm{~d} x\right]^{\frac{1}{2}}<\infty .
$$


iii) For the function $h:[0,1] \times R \times R \rightarrow R$ the following three conditions are satisfied:

1) For each $t \in[0,1], h(t, . .$.$) is continuous.$

2) For each $z \in X_{\frac{1}{2}}, h\left(., z, z^{\prime}\right)$ is measurable.

3) There is a positive number $c_{1}$ such that

$$
\left\|g\left(t, z, z^{\prime}\right)\right\| \leq c_{1}\|z\|,
$$

for all $(t, z) \in[0,1] \times X_{\frac{1}{2}}$.

iv) $I_{k} \in C\left(X_{\frac{1}{2}}, X_{\frac{1}{2}}\right), k=1, \cdots$, and there exist constants $d_{k}, k=1, \cdots, m$, such that

$$
\left\|I_{k}(z)\right\|_{\frac{1}{2}} \leq d_{k}, z \in X_{\frac{1}{2}} .
$$

Here we choose $\alpha=\beta=\frac{1}{2}$. According to paper [21], we know that, if $z \in X_{\frac{1}{2}}$, then $z$ is absolutely continuous , $z^{\prime} \in X$, and $z(0)=z(\pi)=0$. In view of this result, for $(t, z) \in[0,1] \times X_{\frac{1}{2}}, \omega \in \Omega \quad(\Omega$ is defined as in Section 3), we can define respectively that

$$
\begin{gathered}
F(t, z)(x)=\int_{0}^{\pi} b(t, y, x)\left[z(y)+z^{\prime}(y)\right] \mathrm{d} y . \\
G(t, z)(x)=h\left(t, z(x), z^{\prime}(x)\right),
\end{gathered}
$$

and

$$
g(\omega(t))=\sum_{i=0}^{p} K_{i} \omega_{1}\left(s_{i}\right), \omega \in \Omega,
$$

where $K_{i}: X_{\frac{1}{2}} \rightarrow X_{\frac{1}{2}}$ is completely continuous [16] such that $K_{i}(z)(x)=\int_{0}^{\pi} k_{i}(y, x) z(y) \mathrm{d} y$ and $G:[0,1] \times X_{\frac{1}{2}} \rightarrow X \quad$ It is easy to see that $F:[0,1] \times X_{\frac{1}{2}} \rightarrow X_{\frac{1}{2}}, A^{\frac{1}{2}} F:[0,1] \times X_{\frac{1}{2}} \rightarrow X_{\frac{1}{2}} \cdots$ In fact, for each $t \in[0,1]$, we have

$$
\begin{aligned}
& \left\langle F(t, z), \omega_{n}\right\rangle \\
& =\frac{1}{n} \sqrt{\frac{2}{\pi}}\left\langle\int_{0}^{\pi} \frac{\partial b(t, y, x)}{\partial x}\left[z(y)+z^{\prime}(y)\right] \mathrm{d} y, \cos (n x)\right\rangle,
\end{aligned}
$$

also,

$$
\begin{aligned}
& \left\langle F(t, z), \omega_{n}\right\rangle \\
& =\frac{-1}{n^{2}} \sqrt{\frac{2}{\pi}}\left\langle\int_{0}^{\pi} \frac{\partial^{2} b(t, y, x)}{\partial x^{2}}\left[z(y)+z^{\prime}(y)\right] \mathrm{d} y, \sin (n x)\right\rangle .
\end{aligned}
$$

This shows that $\mathrm{F}$ and $A^{\frac{1}{2}} F$ both take values in $X_{\frac{1}{2}}$ in terms of properties (a') and (b'), and therefore the function $g$. Since, for any $x_{1}, x_{2} \in X_{\frac{1}{2}}$,

$$
\begin{aligned}
& \left\|x_{2}-x_{1}\right\|^{2}=\sum_{n=0}^{\infty}\left\langle x_{2}-x_{1}, z_{n}\right\rangle^{2} \leq \sum_{n=0}^{\infty} n^{2}\left\langle x_{2}-x_{1}, z_{n}\right\rangle^{2} \\
& \leq\left\|x_{2}-x_{1}\right\|_{\frac{1}{2}}^{2} .
\end{aligned}
$$

This inequality alongwith condition (ii) says that (H2) is satisfied. Also $G$ satisfies (H3)' and $g$ satisfies (H4). By (i), $F(t, z)$ is a bounded linear operator on $X$. Thus $(H 1),(H 2),(H 3)^{\prime},(H 4),(H 5)$ are satisfied and the system (1) is controllable on $[0,1]$.

\section{Exact Controllability in Infinite Dimensional Space}

It has been observed that the example in $([2-11,22])$ cannot be recovered as special case of the abstract result. If the semigroup is compact then the assumption (H1) in Section 2 is valid only in finite dimensional space so the applications are restricted to ordinary differential control system but not to partial differential equations (refer [15]). We have tried to overcome to this problem in Section 3 for the inclusion (1). Here we present another way of exact controllability result of the system (4) in infinite dimension space.

LEMMA 5.1 Let $\left.\mathcal{P C}([0, \tau]), X_{\alpha}\right)$ be a space formed by normalized piecewise continuous function $\left([0, \tau], X_{\alpha}\right)$. Let $\mathcal{B} \subseteq \mathcal{P C}$ such tha $\tilde{\mathcal{B}}_{k}=\left\{\tilde{V}_{k}: V \in \mathcal{B}\right\} ;$ where

$$
\tilde{V}_{k}(t)=\left\{\begin{array}{l}
V(t) ; t \in\left(t_{k}, t_{k+1}\right] \\
V\left(t_{k}^{+}\right) ; t=t_{k}
\end{array}\right.
$$

The set $\mathcal{B} \subseteq \mathcal{P C}$ is relatively compact if and only if each set $\tilde{\mathcal{B}}_{k}$ is relatively compact in the space $C\left(\left[t_{k}, t_{k+1}\right] ; X_{\alpha}\right)$.

THEOREM 5.2 Assume that the function $F$ and $G$ verify the assumptions $(H 1)$ and $(H 2)^{\prime}$ respectively and suppose that the following conditions are fulfilled:

a) For every $r>0$ and all $\varepsilon>0$ there are compact sets $U_{\varepsilon, r}^{i} \subseteq X_{\alpha}, i=1,2$, such that $T(\varepsilon) A^{\beta} F(s, \xi) \in U_{\varepsilon, r}^{1}$ and $T(\varepsilon) G(s, \xi) \in U_{\varepsilon, r}^{2}$ for every $F(\xi): J \times \mathcal{B}_{r}(0, \Omega)$.

b1) Conditions $(H 3)$ and $(H 4)$ are satisfied. Then there exists a mild solution of the system (3.4).

Proof Consider the system (3.4). As a main portion of the theorem, we prove that $N$ is completely continuous operator. 
The mild solution given in Theorem (3.2) can be splitted up into following four parts:

$$
\begin{gathered}
N^{(1)}(t)=T(t)\left[x(0)-g(x)-F\left(0, x\left(h_{1}(0)\right)\right)\right] \\
+F\left(t, x\left(h_{1}(t)\right)\right) \\
N^{(2)}(t)=\int_{0}^{t} A T(t-s) F\left(s, x\left(h_{1}(s)\right)\right) \mathrm{d} s \\
N^{(3)}(t) z=\int_{0}^{t} T(t-s) G\left(s, x\left(h_{2}(s)\right)\right) \mathrm{d} s+\int_{0}^{t} T(t-s) B u(s) \mathrm{d} s \\
N^{(4)}(t)=\sum_{0 \leq t_{k} \leq t} T\left(t-t_{k}\right) I_{k}\left(x\left(t_{k}^{-}\right)\right), \text {for each } t \in J .
\end{gathered}
$$

Obviously each $N^{(i)}$ is continuous. To prove that $N$ is compact operator we will show separately that $N^{(i)}\left(\mathcal{B}_{r}(0, \Omega)\right)$ is relatively compact in $\Omega$ for every $x \in \mathcal{B}_{r}=\mathcal{B}_{r}(0, \Omega)$.

Step 1: Let $N^{(1)}\left(\mathcal{B}_{r}\right)$ is relatively compact in $\Omega$.

Let $V=N^{(1)}\left(\mathcal{B}_{r}\right)$. Let $T($.$) is uniformly bounded$ on $[0, b]$ and continuous for the norm of the operator in $(0, b]$, we can observe that the sets

$\tilde{V}_{k} \subseteq-T(t) F\left(0, x\left(h_{1}(0)\right)\right)+A^{-\beta} U_{\varepsilon, r}^{1} ;$ it follows that $\tilde{V}_{k}$ is relatively compact for every $t \in\left[t_{k}, t_{k+1}\right], k=0,1,2, \cdots, m$.

Step 2: Let $N^{(2)}\left(\mathcal{B}_{r}\right)$ is relatively compact in $\Omega$.

We first show that $N^{2}\left(\mathcal{B}_{r}(t)\right)$ is relatively compact for each $t \in J$. For $t=0$ it is trivial. Assume that $0<2 \varepsilon<t \leq b$ and let $U_{\varepsilon, r}^{1}$ be the compact set introduced in the hypothesis $(a 1)$. Since $A^{\beta} T($.$) is$ strongly continuous on $[\varepsilon, b]$, it follows that

$U_{\varepsilon}=\left\{A^{1-\beta} T(s) x: s \in[\varepsilon, b], x \in U_{\varepsilon, r}^{1}\right\}$ is relatively compact in $X_{\alpha}$.

Now using mean value theorem for Bochner integral, we can write

$$
\begin{aligned}
& N^{(2)} x(t)=\int_{0}^{t-2 \varepsilon} A^{1-\beta} T(t-s-\varepsilon) T(\varepsilon) A^{\beta} F\left(s, x\left(h_{1}(s)\right) \mathrm{d} s\right. \\
& +\int_{t-2 \varepsilon}^{t} A^{1-\beta} T(t-s) A^{\beta} F\left(s, x\left(h_{1}(s)\right) \mathrm{d} s\right. \\
& \in(t-2 \varepsilon) \overline{\operatorname{co}\left(U_{\varepsilon}\right)}+\left(\mathcal{B}_{r^{*}}\right)\left(0, X_{\alpha}\right)
\end{aligned}
$$

for each $x \in\left(\mathcal{B}_{r}\right)$, where $\operatorname{co}\left(U_{\varepsilon}\right)$ denotes convex hull of $U_{\varepsilon}$ and

$$
r^{*}=2^{\beta} C_{1-\beta}\left\{L_{1}\left(\|x\|_{\alpha}+1\right)\right\} \frac{\varepsilon^{\beta}}{\beta} .
$$

Thus $N^{2}\left(\mathcal{B}_{r}\right)(t)$ is relatively compact in $X_{\alpha}$. Next we show that $N^{(2)}\left(\mathcal{B}_{r}\right)$ is equi-continuous.

Let $0 \leq t_{0}<t \leq b$. Then

$$
\begin{aligned}
& N^{(2)} x(t)-N^{(1)} x(t) \\
&=-\int_{0}^{t} A T(t-s) F\left(s, x\left(h_{1}(s)\right)\right) \mathrm{d} s \\
&+\int_{0}^{t_{0}} A T\left(t_{0}-s\right) F\left(s, x\left(h_{1}(s)\right)\right) \mathrm{d} s \\
&=\left(I-T\left(t-t_{0}\right)\right) N^{(2)} x\left(t_{0}\right) \\
&-\int_{t_{0}}^{t} A T(t-s) F\left(s, x\left(h_{1}(s)\right)\right) \mathrm{d} s
\end{aligned}
$$

Since the elements $N^{(2)} x\left(t_{0}\right)$; for $x \in \mathcal{B}_{r}$; are included in a compact set, it follows that the first term on right hand side converges uniformly to zero as $t \rightarrow t_{0}$. Similarly it follows from $(b 1)$ that the function $A T(t-s) F\left(s, x\left(h_{1}(s)\right)\right), x \in \mathcal{B}_{r}$ are equi-integrable, which imply that the second term on right hand side also converges uniformly to zero as $t \rightarrow t_{0}$.

This show that $N^{(2)}\left(\mathcal{B}_{r}\right)$ is equi-continuous from the right at $t_{0}$. Similarly it can be prove that $N^{2}\left(\mathcal{B}_{r}\right)$ is equi-continuous from the left at $t_{0}>0$. Thus $N^{(2)}\left(\mathcal{B}_{r}\right)$ is equi-continuous and hence $N^{(2)}\left(\mathcal{B}_{r}\right)$ is relatively compact in $\Omega$.

Step 3: By using same argument as in Step 2 we can prove that the set $N^{(3)}\left(\mathcal{B}_{r}\right)$. is relatively compact in $\Omega$.

Step 4: The relatively compactness of $N^{(4)}\left(\mathcal{B}_{r}\right)$. is consequence of assumption (H4) and Lemma 5.1 Hence the proof.

Remark Throughout Section 5 we have used compactness assumption of Theorem 5.2 (a1) and growth condition (H2) (ii) and (H5). If the maps $\mathrm{F}$ and $I_{k}, k=1,2, \cdots, m$ satisfy some Lipschitz conditions (H2) (i) and (H5) instead of compactness in (a1) then also we can prove controllability result.

\section{References}

[1] M. Martelli, “A Rothe's Type Theorem for Noncompact Acyclic-Valued Map," Bollettino dell'Unione Mathematica Italiana, Vol. 2, 1975, pp. 70-76.

[2] M. Benchohra and S. K. Ntouyas, "Existence Results for Nondensely Defined Impulsive Semilinear Functional Differential Inclusion with Infinite Delay," Journal of Fixed Point Theory and Applications, Vol. 2, No. 1, 2007, pp. 11-51.

[3] C. C. Travis and G. F. Webb, "Existence, Stability and Compactnes with $\alpha$-Norm for Partial Functional Differential Equations," Transaction of American Mathematical Society, Vol. 240, 1978, pp. 129-143.

[4] L. Byszewski, "Theorems about the Existence and Uniqueness of a Semilinear Evolution Nonlocal Cauchy Problem," Journal of Mathematical Analysis and Applications, Vol. 162, 1991, pp. 496-505.

[5] K. Deng, "Exponential Decay of Solutions of Semilinear Paprabolic Equations with Nonlocal Initial Conditions," Journal Mathematical Analysis and Applications, Vol. 179, 1993, pp. 630-637. doi:10.1006/jmaa.1993.1373

[6] D. N. Chalishajar, "Controllability of Second Order Semilinear Neutral Functional Differential Inclusions with Non-Local Conditions in Banach Spaces," Nonlinear Functional Analysis and Applications, Vol. 14, No. 1, 2009, pp. 1-15.

[7] D. N. Chalishajar and F. S. Acharya, "Controllability of Semilinear Impulsive Partial Neutral Functional Differential Equations with Infinite Delay," Proceeding of Fourth International Conference on Neural, Parallel Scientific and Computations, Atlanta, 2010, pp. 83-89. 
[8] D. N. Chalishajar and F. S. Acharya, "Controllability of Second Order Semi-linear Neutral Impulsive Differential Inclusions on Unbounded Domain with Infinite Delay in Banach Spaces," Bulletin of Korean Mathematical Society, Vol. 48, No. 4, 2011, pp. 813-838.

[9] D. N. Chalishajar, R. K. George, A. K. Nandakumaran and F. S. Acharya, "Trajectory Controllability of Nonlinear Integro-Differential System," Journal of Franklin Institute, Vol. 347, 2010, pp. 1065-1075.

[10] M. Benchohra and S. K. Ntouyas, "Existence and Controllability Results for Nonlinear Differential Inclusions with Nonlocal Conditions in Banach Spaces," Journal of Applied Analysis, Vol. 1, No. 8, 2002, pp. 45-52.

[11] M. Benchohra and S. K. Ntouyas, "On Second Order Impulsive Functional Differential Equations in Banach Spaces," Journal of Applied Mathematics and Stochastic Analysis, Vol. 15, 2002, pp. 45-52. doi:10.1155/S1048953302000059

[12] E. Hernandez, M. Rabello and H. R. Henriquez, "Existence of Solutions for Impulsive Partial Neutral Functional Differential Equations," Journal of Mathematical Analysis and Applications, Vol. 31, 2007, pp. 1135-1158. doi:10.1016/j.jmaa.2006.09.043

[13] D. N. Chalishajar, "Controllability of Nonlinear IntegroDifferential Third Order Dispersion System," Journal Mathematical Analysis and Applications, Vol. 348, 2008, pp. 480-486. doi:10.1016/j.jmaa.2008.07.047

[14] X. L. Fu and Y. J. Cao, "Existence for Neutral Impulsive Differential Inclusions with Nonlocal Conditions," Nonlinear Analysis-TMA, Vol. 68, 2008, pp. 3707-3718.
[15] R. Triggani, "A Note on Lack of Exact Controllability for Mild Solution in Banach Spaces," SIAM, Journal of Control and Optimization, Vol. 18, No. 1, 1980, pp. 98-99.

[16] K. Yosida, "Functional Analysis," 6th Edition, SpringerVerlag; Berlin, 1980.

[17] K. Deimling, "Multivalued Differential Equations," De Gruyter, Berlin, 1992. doi:10.1515/9783110874228

[18] L. Byszewski, "Existence of Solutions of Semilinear Functional-Differential Evolution Nonlocal Problem," Nonlinear Analysis, Vol. 34, 1998, pp. 65-72. doi:10.1016/S0362-546X(97)00693-7

[19] A. Pazy, "Semigroups of Linear Operators and Applications to Partial Differential Equations," Springer-Verlag, New York, 1983. doi:10.1007/978-1-4612-5561-1

[20] H. Themies, "Integrated Semigroup and Integral Solutions to Abstract Cauchy Problems," Journal Mathematical Analysis and Applications, Vol. 152, 1990, pp. 416-447. doi:10.1016/0022-247X(90)90074-P

[21] C. C. Travis and G. F. Webb, "Partial Functional Differential Equations with Deviating Arguments in Time Variable," Journal of Mathematical Analysis and Applications, Vol. 56, 1976, pp. 397-409. doi:10.1016/0022-247X(76)90052-4

[22] Y. K. Chang and D. N. Chalishajar, "Controllability of Mixed Volterra-Fredholm-Type Integro-Differential Inclusions in Banach Spaces," Journal of Franklin Institute, Vol. 345, 2008, pp. 449-507. doi:10.1016/j.jfranklin.2008.02.002 